\title{
GEOPHYSICAL PREDICTION TECHNOLOGY BASED ON ORGANIC CARBON CONTENT IN SOURCE ROCKS OF THE HUIZHOU SAG, THE SOUTH CHINA SEA
}

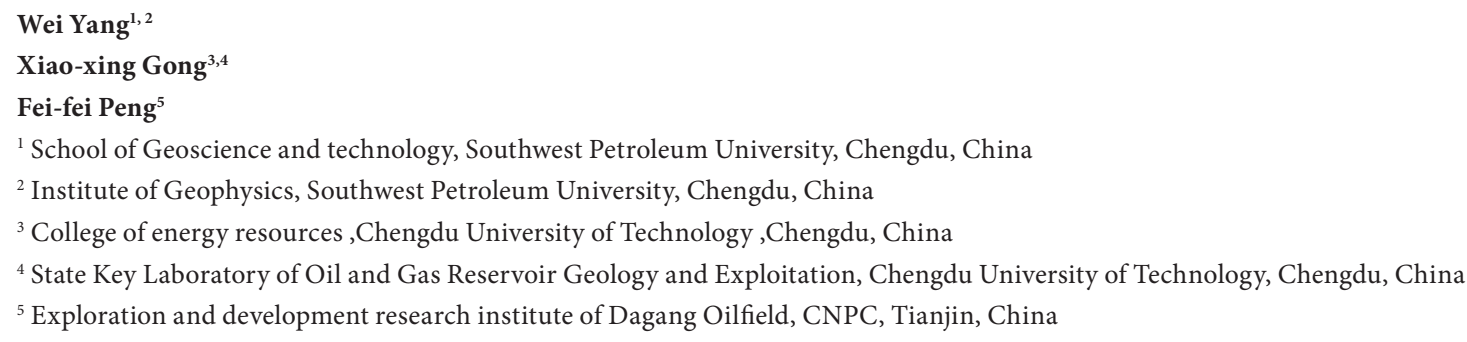

\begin{abstract}
Due to the high exploration cost, limited number of wells for source rocks drilling and scarce test samples for the Total Organic Carbon Content (TOC) in the Huizhou sag, the TOC prediction of source rocks in this area and the assessment of resource potentials of the basin are faced with great challenges. In the study of TOC prediction, predecessors usually adopted the logging assessment method, since the data is only confined to a "point" and the regional prediction of the source bed in the seismic profile largely depends on the recognition of seismic facies, making it difficult to quantify TOC. In this study, we combined source rock geological characteristics, logging and seismic response and built the mathematical relation between quasi TOC curve and seismic data based on the TOC logging date of a single well and its internal seismic attribute. The result suggested that it was not purely a linear relationship that was adhered to by predecessors, but was shown as a complicated non-linear relationship. Therefore, the neural network algorithm and SVMs were introduced to obtain the optimum relationship between the quasi TOC curve and the seismic attribute. Then the goal of TOC prediction can be realized with the method of seismic inversion.
\end{abstract}

Keywords: Huizhou sag; Source rock; TOC; Separate-frequency inversion; Geophysical prediction

\section{INTRODUCTION}

Owing to the high cost of drilling in the offshore basin, most of the prospect wells are placed in the high position of structure where source rocks lack, leading to limited number of wells encountered in the drilling of source rocks, limited proper source rock samples and restricted distribution in the region. Since it costs much to analyze the Total Organic Carbon Content (TOC) of source rocks based on the geochemical method, and the traditional geologic research method cannot obtain continuous TOC of the region, it is difficult to predict on the area of source rocks. It has become a difficulty in offshore basin research how to economically, efficiently and accurately predict source rocks with existing data. The research results show a mathematical relationship between various logging parameters and TOC (Meyer B.L. et al. 1984). An appropriate quantitative prediction model can be established based on this relationship to quantitatively predict the single well TOC of source rocks (Fertl W.H. et al. 1988). The advantage of source rock logging evaluation lies in the definition of the "point". The regional source rock prediction mostly stresses on the recognition of seismic facies in sedimentary facies that mainly develop dark mudstones and shale. Not all dark mudstone and shale will turn to effective source rocks that are rich in organic carbon and contribute to hydrocarbon reservoirs. The advantage of the source rock prediction in seismic facies lies in that it can roughly confirm the area rich in source rock plane distribution, but it cannot quantitatively and precisely predict regional TOC. The 
geophysical inversion method combined with logging-seismic data is currently seldom adopted in the regional quantitative prediction of TOC.

In this article, firstly, the geophysical responses characteristics of source rocks are quantified based on the advantage of logs' longitudinal magnification in "point" (Herrson S.L. 1988). Then, the relationship between the TOC of testing samples and logs is established (Mann U.P. et al. 1988). At the same time, the well-seismic relationship is employed to transform the seismic data from the "point" into the "line", to attain the goal of predicting the regional TOC.

\section{THE GEOLOGICAL CHARACTERISTICS OF SOURCE ROCKS IN THE HUIZHOU SAG}

The Huizhou sag is located in the middle area of the $\mathrm{Zhu}$ I Depression in the Pearl River Mouth Basin. The Zhu I Depression, which lies in the northern depression, develops as a slim sag that is distributed in the NE direction (Fig. 1).

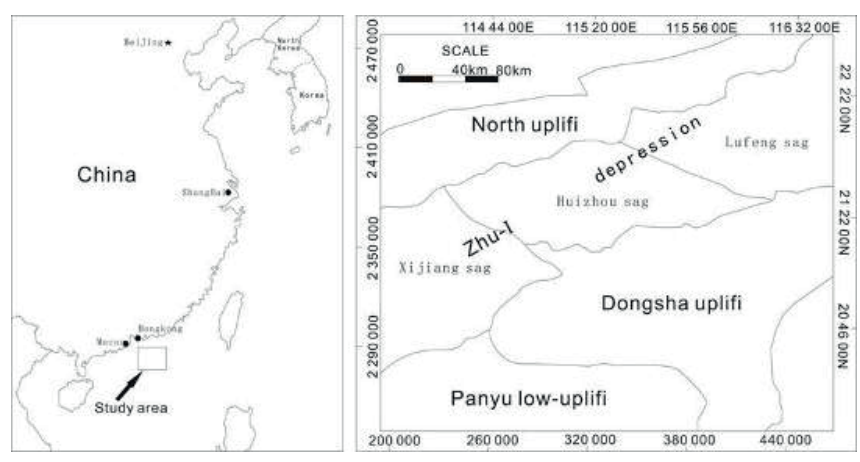

Fig.1. The Location and Structure Map of the Study Area

The Pearl River Mouth Basin is an extensional basin fractured in late Cretaceous, and the result of the expansion of the Southern China Sea. The Huizhou sag mainly develops two groups of effective source rocks, including the Eocene Wenchang Formation and Eocene-lower Oligocene Enping Formation. During the fault-subsiding period of the deposited Wenchang Formation, it is featured with a depositional environment of shallow and mid-deep lacustrine facies and lacustrine-shore and lithological characters mainly of a set of thick dark mudstone. The source rocks are mainly distributed in the southwest and east of the Huizhou sag, with the maximum thickness of the Wenchang Formation in sag reaching $1,000-2,000 \mathrm{~m}$, where the proportion of mid-deep lacustrine dark mudstone exceeds $60 \%$, compared to others that occupy less than $40 \%$. The Enping Formation deposited mostly in an environment of shallow lacustrine or fluvial or delta-plane facies, while it is more extensively distributed than the Wenchang Formation, with a maximum thickness between $1,100-1,600 \mathrm{~m}$ in the sag. It mainly consists of silt and sandstone, intercalated with coal seams and carbon-bearing mudstone, with the grain coarser in an ascending order. The thickness of source rocks is $140-800 \mathrm{~m}$, and the proportion of dark mudstone ranges between 20 and 30\% (Gong Zai Sheng et al.2004). According to the preparatory evaluation of oil and gas resources, the hydrocarbon generation of source rocks which developed from the mid-deep lacustrine dark mudstone in the Wenchang Formation is greater than that from the lacustrine-paludal source rocks in the Enping Formation. The Wenchang Formation is the main source of hydrocarbon in this area.

\section{ANALYSIS OF THE GEOPHYSICAL RESPONSE OF SOURCE ROCKS}

\section{THE RESPONSE CHARACTERISTICS OF THE SOURCE ROCK LOGS}

The difference of the organic carbon content and the fluid in source rocks will definitely lead to the difference of the log response characteristics. Therefore, the effective source rocks can be identified through the difference of the log response (Beers R.F. 1945).

Logging identification and evaluation of hydrocarbon in the source rocks is based on the different logging curve response of organic carbon content and the physical properties of filling-pore fluid. Generally, source rocks that are rich in organic carbon are characterized by low density and strong adsorption characteristics. Source rocks rich in organic carbon are composed of rock matrix, solid organic matter and pore fluid, while the non-source rocks are only composed of rock matrix and pore fluid (Fig. 2A). The pore space of immature source rocks is only filled with the formation water (Fig. 2B), but mature source rock converted part of organic materials into liquid hydrocarbon that fill in the pores, with its space pore filled with both the formation water and liquid hydrocarbon (Fig. 2C).

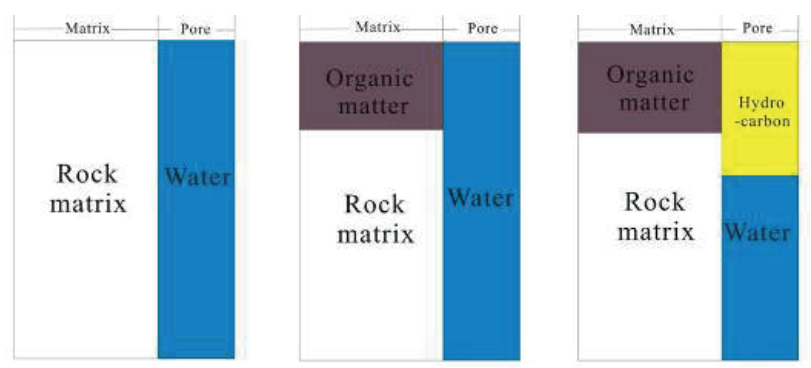

A Non-source rock B Immature source rock C Mature source rocks Fig. 2/ Hydrocarbon Source in Rock Model

The research reveals the following logging response characteristics of source rocks:

1) Radioactivity of source rocks

Source Rocks generally contain rich shale content and organic materials. As the mud enjoys strong adsorption ability for radioactive elements, the uranium content and organic content in the source rock strata hold a positive correlation, with the natural gamma ray spectrometry value 
higher than non-source rocks. The higher natural gamma ray spectrometry and the uranium content are employed to identify the source socks (Schmoker J.W. 1981).

\section{2) Conductivity of source rocks}

The mudstone is featured with a good electrical conductivity (as the rock matrix and pore water layer are all electrically conductive), resulting in low resistivity of the strata (excluding calcareous strata). However, the mudstone layer richer in organic materials holds a resistivity always smaller than the same types of formation without organic materials, due to kerogen, oil and gas with poor conductivity.

\section{3) Density of source rocks}

Since organic materials are low in density $\left(\right.$ about $1 \mathrm{~g} / \mathrm{cm}^{3}$ ), compared to the density of the framework of clay minerals reaching approximately $2.7 \mathrm{~g} / \mathrm{cm}^{3}$, the formation density will decrease when the source rocks replace the rock skeleton.

\section{4) Interval transit time of source rocks}

Generally, the interval transit time of mudstone and shale decreases along with the increase of its burial depth (when the formation compaction degree increases). However, in the case that the formation contains organic materials or oil and gas, the greater content of organic materials in the rocks will lead to greater interval transit time since the P-wave velocity of organic materials is significantly smaller than the rock skeleton.

Based on the logging response characters of 178 geochemical tested samples that achieve the standard of effective source rocks of the Wenchang formation in the Huizhou sag, the logging response of source rocks in the Wenchang formation is presented as "three medium and one low": medium AC: 70-90, medium GR: 50-90, medium density: 2.5, and low SP: about -15 left and right.

\section{SEISMIC RESPONSE CHARACTERISTICS OF SOURCE ROCKS}

The seismic line crossing LF-A well is located in the east half graben of the Huizhou sag, where the maximum thickness of the Wenchang Formation is up to $1,800 \mathrm{~m}$. The Wenchang formation at the LF-A well is $92 \mathrm{~m}$ thick, mainly composed by gray tuffaceous shale and siliceous shale, with a small amount of fine sandstone and muddy sandstone at the bottom. From bottom to top, the strata becomes finer and finer, while the overall environment is constituted of the semi-deep lacustrine facies. The measured TOC value of the well shows that the Wenchang formation of dark mudstone in the deep lacustrine facies consists of effective source rocks.

From the seismic profile of the LF-A well (Fig. 3), we can see the seismic facies of deep lacustrine facies, dark mudstone source rocks are featured with low frequency and continuous strong reflection. The lower section of the sag of the LF-A located well is corrugated based on the low frequency, intermittence and low amplitude reflection, belonging to the shore shallow-deep lacustrine facies, while the upper presents the sub parallel/parallel low frequency, continuity and high amplitude reflection, mainly suggesting the deep lacustrine deposition. It shows that the water body becomes deeper from bottom to top and the sedimentary environment transforms from fluvial facies to the shallow lacustrine facies - semi deep lacustrine facies. The disorder reflection at the fault edge place near the NW direction shows a small Delta deposition. Based on the characteristics of seismic facies, the medium and deep lacustrine facies of "low frequency, continuity and high amplitude reflection" in the subsag is extensively and continuously distributed. The medium and deep lacustrine facies with the above reflections are widely distributed in the Huizhou depression, while the seismic response characters reflect that the Huizhou sag enjoys favorable sedimentary conditions for the development of effective source rocks.

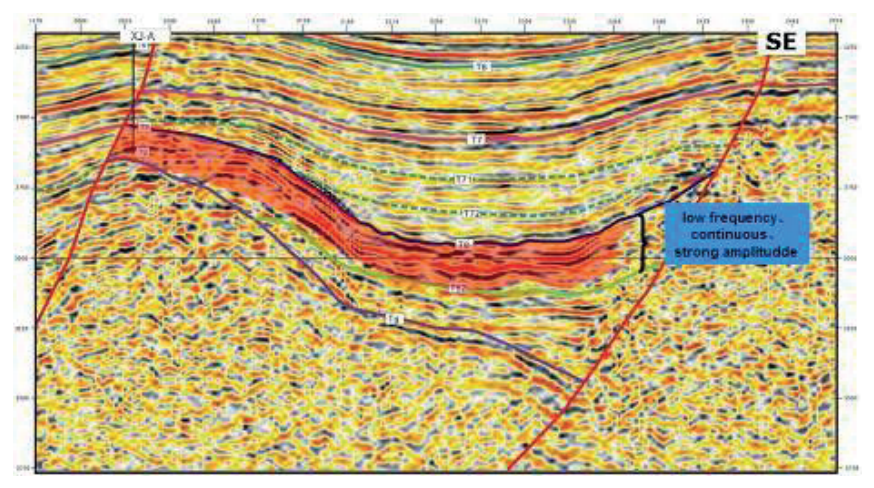

Fig. 3. Seismic Response Characteristics of Source Rocks Crossing XJ-A Seismic Line

\section{PREDICTION METHODS OF SOURCE ROCKS}

\section{TECHNICAL IDEAS}

The geophysical prediction method of the organic carbon content, means to establish the mathematical relation between the measured organic carbon content value and the different logging data based on mathematical algorithms, mainly based on the Total Organic Carbon Content (TOC) value tested by a few single wells in source rocks, apply the mathematical relationship to evaluate and predict the organic carbon content of source rocks in other wells not having been measured (Herron S.L. 1987). The advantages of TOC evaluation method of the logging source rocks lie in the accurate prediction of TOC values, but the method can only be adopted to well points and is difficult to predict source rocks in regional area.

Regarding the organic carbon content seismic prediction, the commonly used method was to directly establish the linear relationship between the measured TOC value and GR value, then conduct the acoustic curve fitting through the GR curve, establish the relationship between the pseudo-wave impedance and TOC value, and finally complete the inversion of source rocks TOC profile. Although it is rapid and direct, this method also reveals evident disadvantages. Firstly, the direct linear relationship between the measured TOC value 
and GR does not exist in many cases, which will result in the low accuracy of the base value before inversion extrapolation and thus lead to the non-accuracy of the inversion results. Secondly, this method directly establishes the reconstruction of the acoustic curve and considers that there is a simple linear relationship between the wave impedance and TOC value. However, in the actual research done by the author, the linear correlation between the impedance value and the TOC value is found to be low. Then, the TOC value directly obtained through the linear solution from the original seismic data is not as true as predicted.

During the research on the geophysical prediction of the source rocks in the Huizhou sag, the technical routes have been formed as below (Fig. 4).

1) Based on the log response characteristics of source rocks, apply multiple logging curves to establish several mathematical relationships between the few measured TOC values and logging curves and build the quasi TOC curve of a single well.

2) Extract various seismic internal attributes from the original seismic profile of the borehole side, including instantaneous frequency, time frequency, filtering, derivative, integral, time and other property types, and compare the seismic internal attributes to the quasi TOC curve correlation, and select the dominant seismic internal attributes.

3) Two following methods are respectively applied in the TOC value prediction of seismic inversion.

Method I: Select multiple attributes from the seismic internal attributes that are the most relevant to the quasi TOC curve. Apply the neural network algorithm to establish mapping between seismic attributes and quasi TOC curve. Finally, the inversion prediction on TOC is made through the relationship based on the original seismic data.

Method II: Establish the relationship between the quasi TOC curve and a different frequency-band attribute, and build the non-linear mapping relationship between the frequency attributes produced upon the original seismic data and the quasi TOC curve based on the algorithm of SVMs, and conduct inversion forecast of the TOC value.

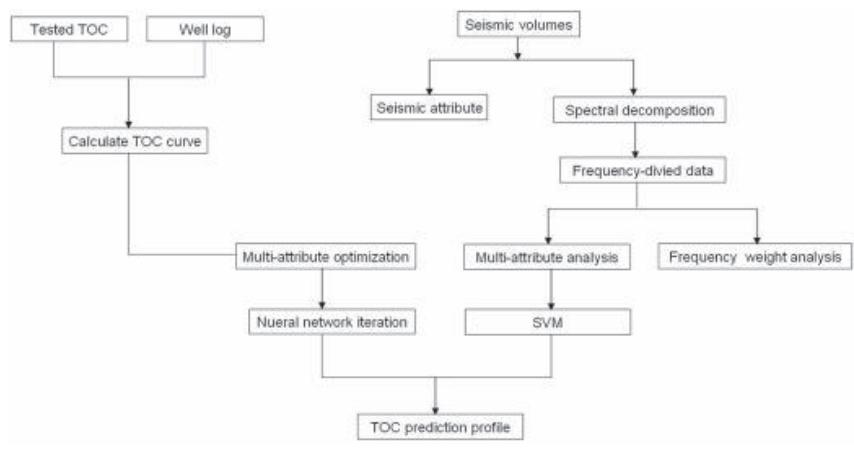

Fig.4. Geophysical Prediction Technology Roadmap of Organic Carbon Content Value

\section{CALCULATION METHOD OF THE QUASI TOC CURVE}

\section{1) Natural gamma ray spectrometry method}

Compared to the sandstone stratum, the sand-shale stratum is featured with stronger radioactivity and displays a higher numeric value in GR, no matter whether it contains organic materials or not. If the radioactivity of the strata is determined by the shale content in the formation, the natural gamma ray spectrometry curve often corresponds to higher $\mathrm{TH}$ content, when the abundance of the radioactive $\mathrm{U}$ element is lower than TH. If the high radioactive formation is based on the presence of organic materials, the $U$ content in the formation will increase, compared to the lower abundance of $\mathrm{TH}$, due to the proportional relation between the radioactive nuclides of $U$ and the organic content. Therefore, we can define a parameter to describe the relative abundance of $U$ and $\mathrm{TH}$ in the strata, which is called U/TH (FERTL W.H. et al. 1980).

Natural gamma ray spectrometry, i.e. the gap between the total GR and excluding-U GR, not only provides the abundance of 3 radioactive nuclides series in the strata (potassium, uranium and thorium content), but also provides the counting rate of total GR and excluding-U GR in the formation. The difference between the total GR curve and excluding- $U$ GR is related to the content of $U$ in the strata, while the greater difference suggests the higher content of $U$ in the formation, and vice versa. According to the relationship between the uranium content and organic materials, it can be inferred that the difference of two gamma curves is related to the organic content in the strata. The difference is denoted as:

$$
\Delta \mathrm{GR}=\mathrm{SGR}-\mathrm{CGR}
$$

Where:

SGR - Total GR value;

CGR - Excluding-U GR value.

In order to eliminate the effect of borehole enlargement, $\Delta \mathrm{GR} / \mathrm{SGR}$ and $\Delta \mathrm{GR} / \mathrm{CGR}$ can also be used. Obviously, the higher the content of the radioactive nuclides of $U$ in the formation the greater numerical value of $\Delta \mathrm{GR}$ and $\Delta \mathrm{GR} / \mathrm{CGR}$. In case of a large amount of rich-potassium minerals in the formation, the total radioactivity is higher in the strata, and the counting rate of excluding-U GR is higher; correspondingly, the $\Delta \mathrm{GR}$ is lower.

The 4,250m-4,320m section of the HZ-A well has SGR and CGR logging curve. The $\Delta$ GR / SGR curve upon the fitting of the SGR and CGR curve can reflect the variation of organic carbon content in source rocks in the formation. A linear relation can be established based on the fitting curve and the organic carbon content in the measured core sample value, i.e.

$$
\mathrm{TOC}=\Delta \mathrm{GR} / \mathrm{SGR} \times \mathrm{R}^{2}+0.146(\mathrm{R}=0.852)
$$

Then, the linear relationship can be applied to calculate TOC values, as shown in Fig. 5. The TOC value curve and the quasi TOC curves obtained are highly related to the measured TOC values. The thickness of the high-quality source rocks can be categorized according to the high-quality 
source rock classification standard of the offshore basins in case of TOC $\geq 3$, and the thickness of the high-quality source rocks reach $34 \mathrm{~m}$.

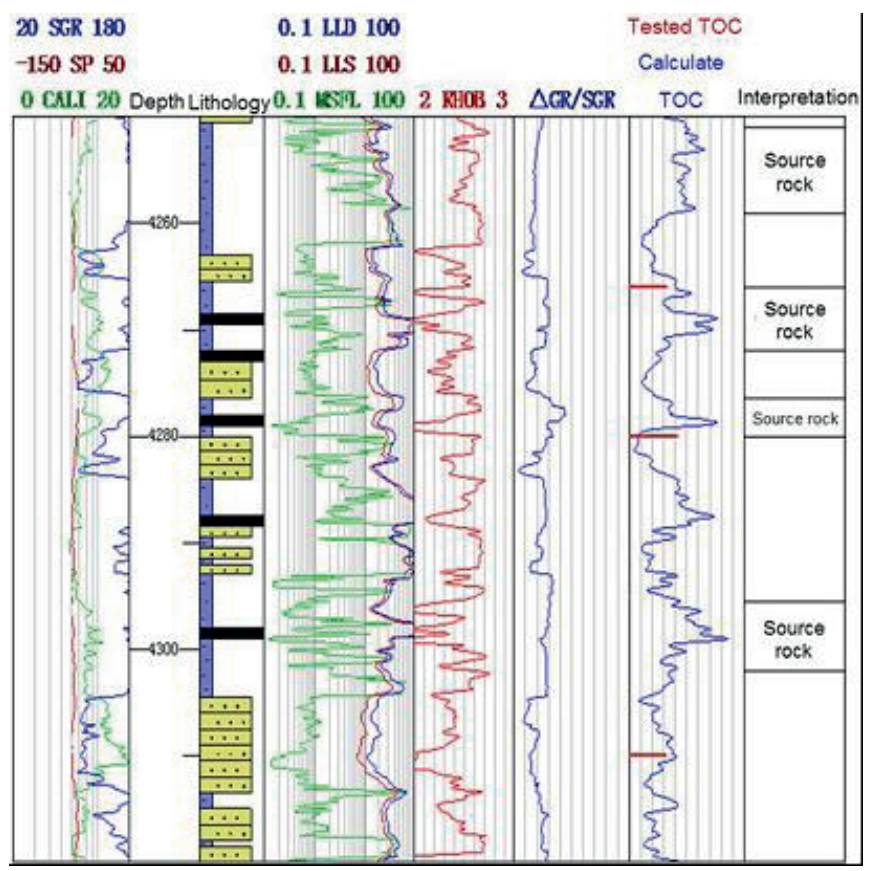

Fig. 5. $\Delta$ GR / SGR Evaluated Method of Source Rock for the HZ-A Well

\section{2) $\Delta \log \mathrm{R}$ Technology}

Passey et al. proposed a logging evaluation method of source rocks, which can calculate the TOC value under different mature conditions (Mohammad Reza Kamalia et al. 2004). This method overlaps the acoustic logging curve of the arithmetic coordinate and resistivity curve of the logarithmic coordinate, and the two curves can be considered as the baseline when both reach "consistent" at a certain depth. After the determination of the baseline, the distance between the two curves on the coordinate of resistivity log is read as $\Delta \log \mathrm{R}$, which is the key point of the $\Delta \log \mathrm{R}$ technique application for the identification of effective source rocks. Its characteristics of logging response indicate that the interval transit time and resistivity should not overlap, shaped like a discontinuous triangular unit in the vertical direction (Passey Q.R. 1990).

In the method that calculates the organic carbon of source rocks based on overlapping log curve of resistivity and interval transit time, the following formula is applied to calculate $\Delta \log R$ :

$$
\Delta \log \mathrm{R}=\lg \left(\mathrm{R} / \mathrm{R}_{\text {baseline }}\right)+0.02\left(\Delta \mathrm{t}-\Delta \mathrm{t}_{\text {baseline }}\right)
$$

Where:

$\Delta \operatorname{logR}$ : The logging curve gap on the coordinate of resistivity; R: Resistivity, $\Omega \bullet m$;

$\Delta \mathrm{t}$ : Value of acoustic travel time, $\mu s / f t$;

$\mathrm{R}_{\text {baseline }}$ : Resistivity value of non source rock baseline corresponding to the $\Delta \mathrm{t}_{\text {baseline }}$
As shown by the overlapping curve of the LF-A well (Fig. 6), the neutron and sonic curves coincide well in the non source rock section, and the overlapping section of two logging curves of the $3,110 \mathrm{~m}-3,140 \mathrm{~m}$ section of the LF-A well constitutes the baseline. The $\Delta \log R$ and TOC value can be calculated through the above formula, for the fitting of the relationship between the measured TOC of the LF-X well and $\Delta \log R$.

$T O C=\Delta \log R \times 10^{2.298-0.1665 R o}$ (Ro: maturity of organic matter)

According to the calculation error analysis between the organic carbon content of measured samples and the TOC, the calculated values agree well with the measured values of the LF-X well, with the errors ranging from $2.23 \%$ to $11.22 \%$, which reflects the high reliability of the calculation. When TOC $\geq 3$, the curve of interval transit time and resistivity do not overlap, but shaped like a discontinuous triangular unit in the vertical direction, featured with a response character of a high organic carbon content value. It reflects that with greater content of organic carbon, the response of logging data to logging source rocks become more significant, demonstrating a better response to high-quality source rocks (effective source rocks). Based on the evaluation of the highquality source rocks upon the calculated TOC value, the high-quality source rocks of LF-X enjoy a thickness up to $26 \mathrm{~m}$.

0.1 LLS $100 \quad 0$ TOC 10

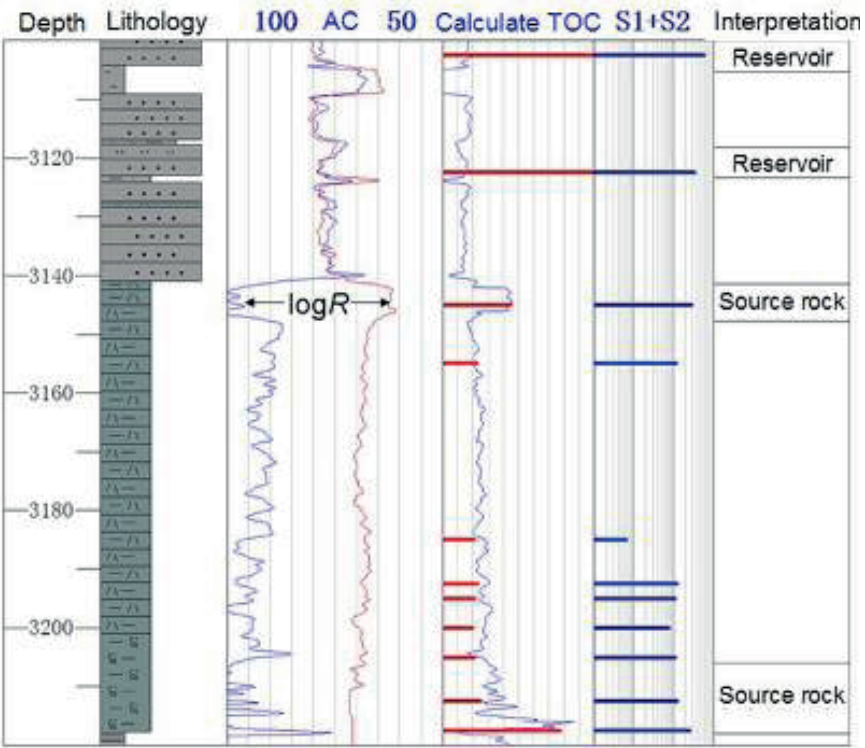

Fig.6. $\triangle G R / S G R$ Evaluated Method of Source Rock for the LF-X Well

3) Solving the multiple regression formula of TOC value

A mathematical mapping relationship exists between the organic carbon content in source rocks and various logging parameters, which enables the more precise quantitative prediction of organic carbon from single logging data. Previously, various simple equations, bivariate equations or variable multiple regression equations have been proposed as the empirical quantitative mode with the total organic 
carbon content as dependent variables, and with single or multiple logging parameters such as the $U$ and $U / K$ ratio, density, neutron porosity, interval transit time and resistivity as independent variables. This method overcomes the shortcoming that a logging curve singly responds to physical properties of the source rocks and more comprehensively takes physical properties of rocks into consideration. In the practical research, we analyze the cross correlation of logging parameters and the TOC value of a measured sample, take into account the influence of the weight index on each logging curve and TOC value, integrate the natural energy spectrum method and Log $\mathrm{R}$ method, thus establish the multiple regression equations of the organic carbon content in the source rocks of a single well and the interval transit time (AC), density (DEN), $\Delta \mathrm{GR} / \mathrm{SGR}$ and $\Delta \log \mathrm{R}$, calculate the TOC value as the dependent variable, consider the four logging parameters as independent variables and solve the multiple regression equations of TOC value:

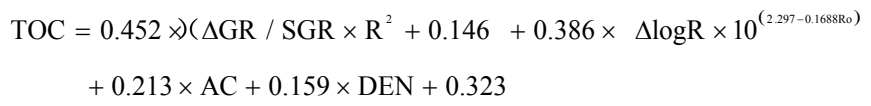

(A, B, C, D and E are constants)

By solving the multiple regression equation, it can avoid the uncertainty in the TOC value calculation based on a single logging curve, effectively improve the correlation between the logging curve and forecast of TOC value and enhance the prediction accuracy of the TOC curve.

In practical application, the author measured the organic carbon content value, the interval transit time (AC), the density (DEN), $\Delta \mathrm{GR} / \mathrm{SGR}$ and $\Delta \log \mathrm{R}$ in the same depth of 113 samples from 6 wells, and conducted a multiple regression analysis from a single parameter to multi parameters and multi compositions. Compared with the single parameter, the forecast of the organic carbon content based on the fitting of four parameters is more accurate, which raised the correlation coefficient of the organic carbon content prediction and the measured values from 0.72 (based on a simple parameter) to 0.78 and lowered the error standard from 1.72 to 1.52 . This is the optimum method to predict the organic carbon content of a single well.

\section{MULTI-ATTRIBUTE INVERSION METHOD FOR TOC VALUE PREDICTION}

\section{1) Method and Principle}

The previous seismic prediction of the TOC value believed that there was a direct linear relationship between the TOC value and acoustic curves. In most cases, the TOC curve was directly transformed into the pseudo acoustic curve, and then the TOC value was predicted through the inversion of the original seismic data into the pseudo wave impedance profile (Russell B.H. 2004). Based on the crossplot of the quasi TOC curve of the single well and the impedance (Fig. 7), it is discovered that the TOC value and wave impedance show low linear correlation, so the previous method could result in a significant prediction error (Fu L.Y. 2004).

During this research, we selected the seismic multiattributes inversion method to predict the TOC value, which is a process for transforming the seismic internal attributes into the lithologic character curve. The seismic internal attributes mainly include the instantaneous attributes, time-frequency attributes, filter sections, derivative, integral property and time (linear gradient) etc. (Robinson E.A. 1957; Robinson E.A. 1980). Firstly, we selected the seismic attributes that present best fitting advantages with the quasi TOC curve, then, applied the neural network algorithm to establish the non-linear relationship between various selected attributes and the quasi TOC curve, utilized this relationship to extrapolate seismic attributes, and finally got the prediction of the TOC profile.

\section{2) Application}

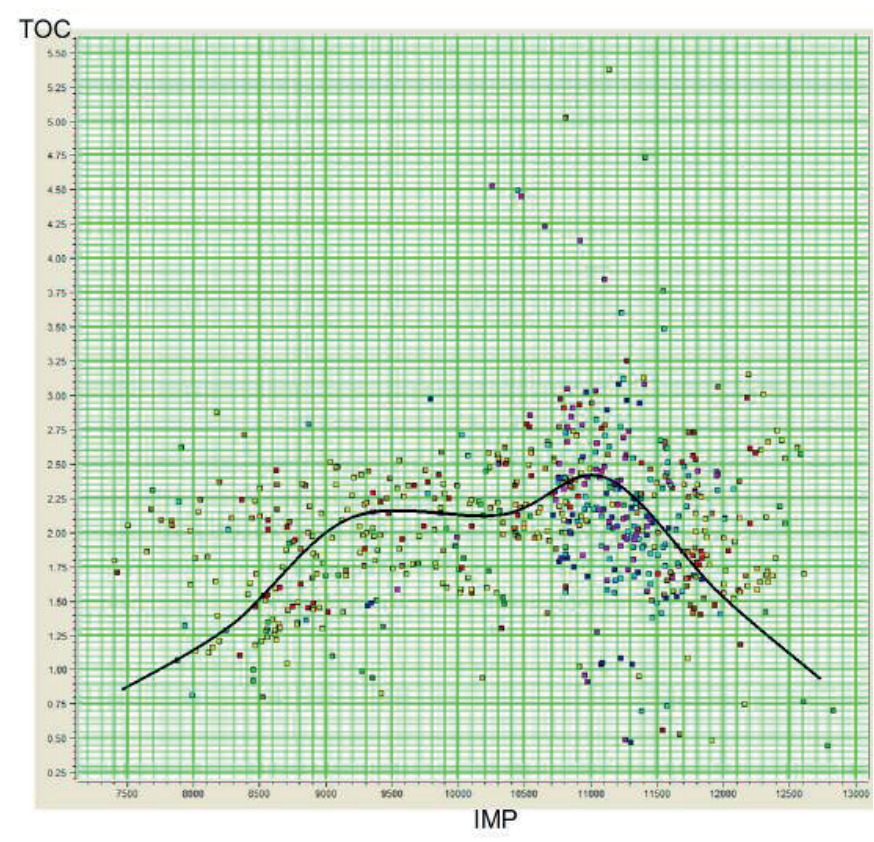

Fig. 7. Crossplot of the Quasi TOC Curve and the Wave Impedance Value

Regarding the selection of seismic attributes, the crossplotted correlation recognition should be conducted for the quasi TOC curve and multiple attributes generated from the seismic trace by well. Different seismic attributes have different physical significance and also show differences in the response of predicted characteristic curve, so it requires repeatedly extracting the seismic attributes and calibrating the relationship errors, to find out the optimum properties. The single attribute presents a relatively single response to the characteristic curve, while the combination of multiple attributes can improve the correlation with the quasi TOC curve. The optimal combination of multiple attributes can be determined with the optimization of the training process (Fig. 8). The optimal combination of seismic attributes consists of the amplitude envelope, 25/30 - 35/40 filter section, average frequency and trace integration. 
When the relation between the combination of seismic multi-attributes and the quasi TOC curve is established, the multivariate regression algorithm and neural network algorithm (Simon Haykin 1999; Lim J.S. 2005) are generally adopted. The multiple regression algorithm directly applies the linear weighted model to acquire the linear relationship between the predicted characteristic curve and seismic multiple attributes. However, the seismic attributes combination and the predicted characteristic curve do not simply constitute a linear relationship, but a complex nonlinear relationship. During the practical research, we applied the neural network algorithm and established the non-linear mapping relationship between seismic attributes and quasi TOC curve through independent learning. Then, after the fitting based on the neural network algorithm, the correlation between the borehole side seismic attributes and quasi TOC curve attains 0.87 , with an average error of $0.59 \%$. The obvious consistency suggests the effectiveness of this method.

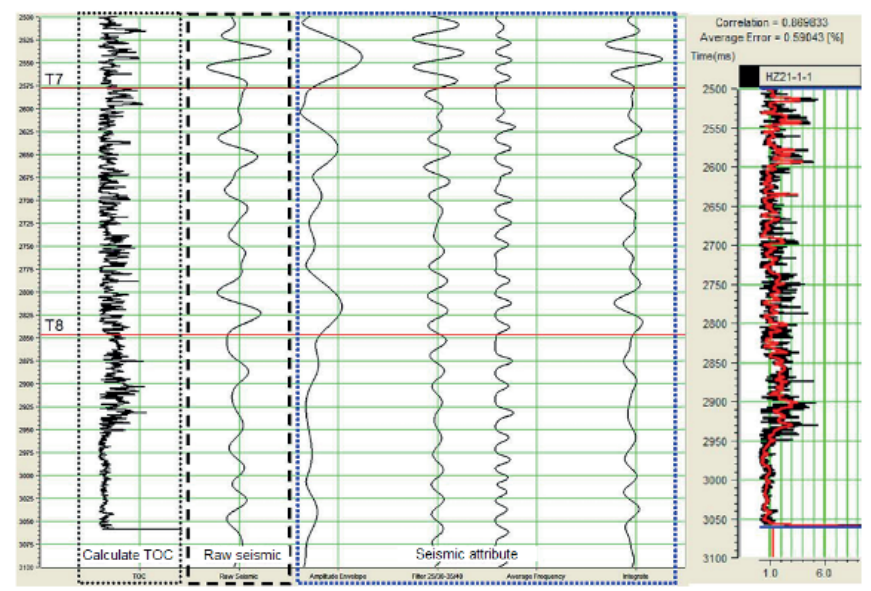

Fig. 8. Seismic Multi-attribute Selection and Fitting Results

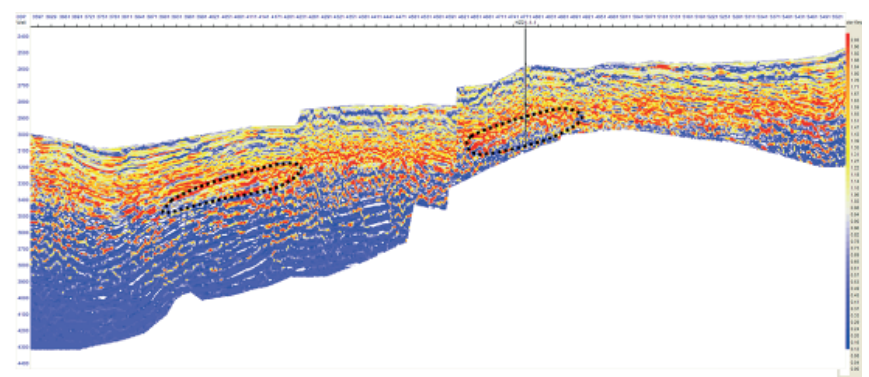

Fig. 9. Multi Attribute Inversion Prediction of TOC Profile Crossing HZ-A

As shown in Fig. 9, the TOC frequency-divided inversion profile crossing the HZ-A well reflects that the organic carbon content of the deep lacustrine facies in the Wenchang formation is higher than that of the upper part of the Enping formation, the TOC value of the Wenchang formation ranges from 2-3.5\%, and the Wenchang formation develops most of the hydrocarbon. The set of effective source rocks are steadily and continuously distributed, with great potential of resources.

\section{FREQUENCY-DIVIDED INVERSION METHOD FOR TOC PREDICTION}

\section{1) Method and Principle}

Seismic data has certain frequency bandwidth, and the seismic trace gathering contains high, medium and low frequency components. For a wedge model, a series of synthetic seismic profile can be obtained from the differentfrequency wavelet deconvolution, so as to acquire the curve of amplitude and thickness at different frequencies, as shown in Fig. 10-left. Transforming Fig. 10-left, you can get various relationships between the frequency and amplitude in different time thickness (AVF) as shown in Fig. 10-right.

By studying the relationship between the amplitude of vibration and frequency of different thickness (AVF), the AVF can be introduced into the inversion as independent information. The rational utilization of the seismic information of high, medium and low frequency band can reduce the uncertainty of the inversion of the thin layer and obtain an inversion result in a high resolution. The frequencydivided inversion is firstly applied to form the frequency bodies, then adopts the SVM technology to establish the mapping relationship between the division and the curve of the target well, and then changes the frequency into characteristic curve through inversion. The advantage of frequency-divided inversion lies in no extracting wavelet during the establishment of the relationship between amplitude and frequency of different thickness strata, not depending on the initial model and the non-linear inversion with high resolution (Zeng Hongliu et al. 2000).

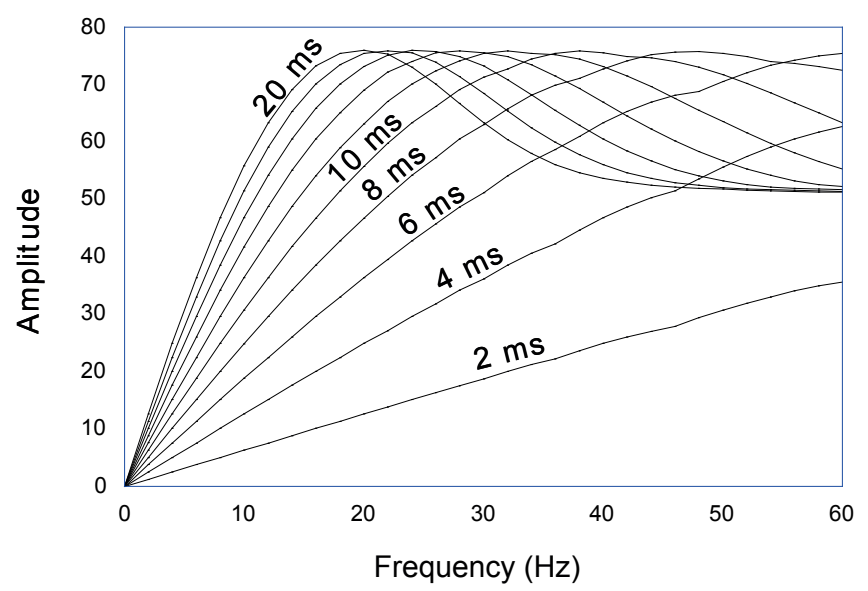

Fig. 10-left Tuning Curve of the Relationship Between the Amplitude and Time Thickness at Different Frequencies 


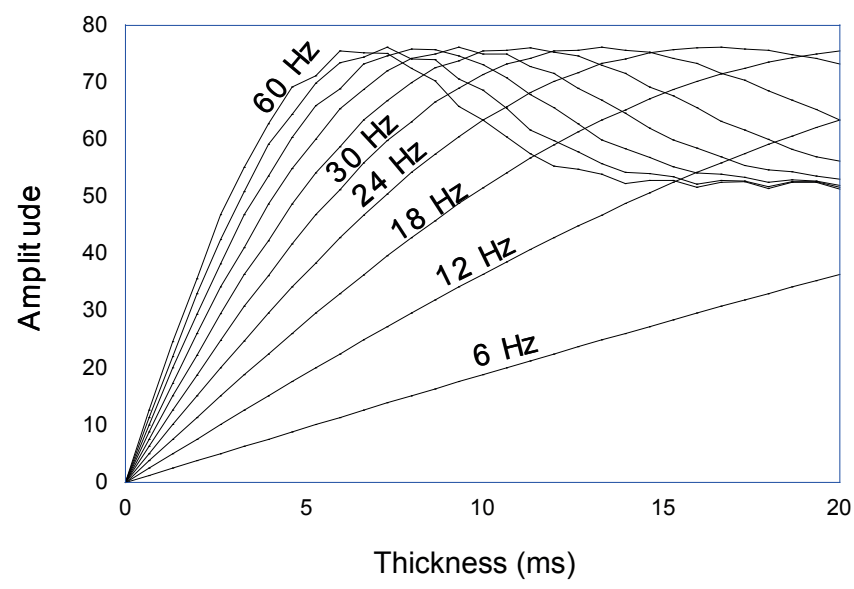

Fig. 10-right Amplitude and Different Frequencies at Time Thickness

\section{2) Application}

1. The effective frequency range can be determined through the analysis of the frequency of seismic profile crossing the LF-A well (Fig. 11-left), showing that the seismic profile gathers three main frequency components, $20 \mathrm{HZ}, 30 \mathrm{HZ}$ and $50 \mathrm{HZ}$.

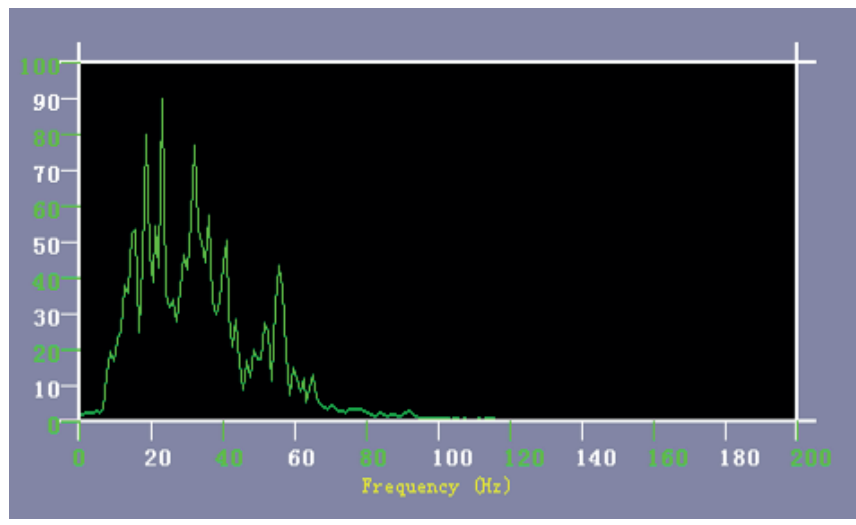

Fig. 11-left. Analysis of the Seismic Profile and Dominant Frequency of Semideep Lacustrine Facies Crossing the LF-A Well

2. The wavelet frequency division technology is employed to complete the frequency division of the original seismic data, which obtains three bands of divided bodies and extracts its instantaneous attributes.

3. The Quasi TOC curve is processed by median filtering. Being a kind of non-linear signal processing technology that inhibits noise, this method will protect the edge signal of the TOC curve, eliminate sharp noise, effectively realize smoothing processing and meanwhile maintain vertical resolution of the quasi TOC curve after filtering.

4. Main stages of source rocks are featured with low frequency and continuous and high-amplitude reflection. According to the frequency analysis on the deep lacustrine facies with the above reflection characteristics in seismic profile, the frequency of source rocks reaches $20 \mathrm{HZ}$ (Fig. 11-right). When establishing the relationship between the spectrum attributes and the quasi TOC value curve, the weight influence coefficient of the $20 \mathrm{HZ}$ frequency attributes is increased to highlight the TOC value response characters and enhance the accuracy of TOC prediction.

5. The SVM algorithm is employed to calculate the relationship between the amplitude and frequency of the different thickness based on the seismic attributes after frequency division. The relationship is introduced into the inversion to establish a non-linear mapping relationship between the pseudo TOC curves and seismic waveform. Eventually, after the introduction of the frequency attributes, the mapping relationship between the body and the TOC prediction target based on SVM is finally adopted to form the inversion body of the TOC value.

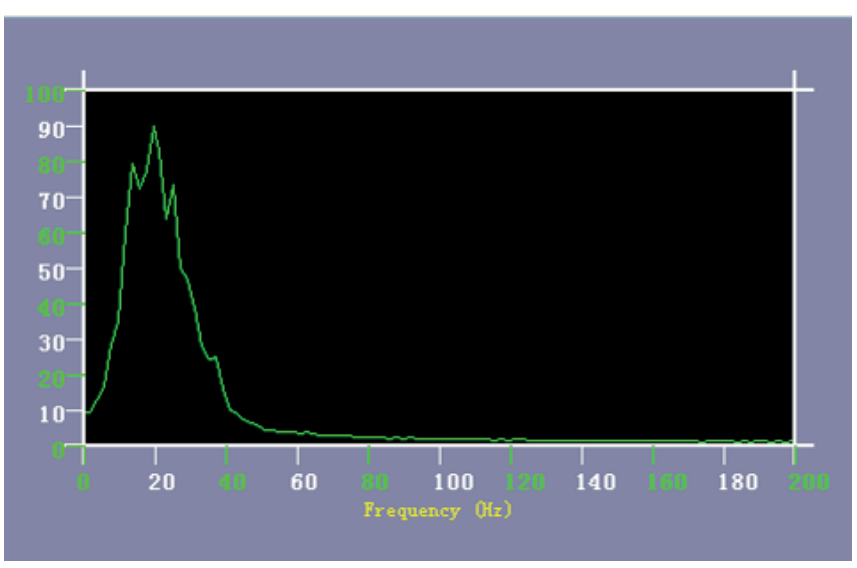

Fig. 11-right. Analysis of the Seismic Profile and Dominant Frequency of Semideep Lacustrine Facies Crossing the LF-A Well

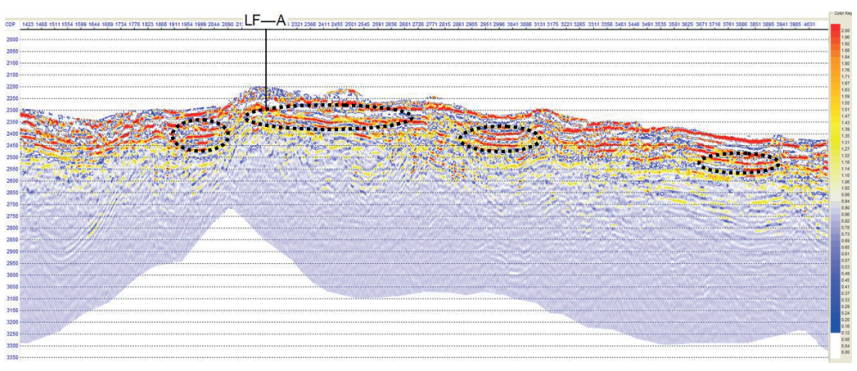

Fig. 12. TOC Frequency Inversion Profile Crossing the LF-A Well

As shown in Fig. 12, according to the profile of the frequency-division inversion prediction of TOC value of the LF-A well, the Wenchang formation contains comparatively higher content of organic carbon, while the overall set of effective source rocks are continuously distributed in the region. The TOC value is higher at the deep sag of the seismic profile and exceeds 2 . It reflects that the sag is the center for the generation of hydrocarbon, featured with high TOC values. 


\section{CONCLUSION}

In the evaluation process of source rocks in the offshore basin of the Huizhou sag, we established a whole set of TOCbased geophysical prediction method, from the measured TOC value of samples, the calculation of the quasi TOC value curve and the seismic inversion of TOC value, which realized the stepwise prediction from "kind", to "point" to "line".

1) Summarize the characteristics of source-rock logging and seismic response based on the analysis of the physical characteristics of source rocks.

2) Several logging curves are introduced during the calculation of the quasi TOC value curve to establish the multiple regression relationship, thus to acquire more accurate evaluation of the TOC value.

3) During the seismic inversion of the quasi TOC curve, a complicated non-linear relationship is found between the TOC value and seismic attributes, so the algorithm of neural network and SVM algorithm can better fit the correlation between the two. The multi-attribute inversion and the frequency inversion method are respectively employed to conduct the TOC value inversion.

4) The geophysical prediction makes up for the limited quantity of sample, ensures the economic and rapid TOC value prediction, offers more accurate and quantitative prediction of the seismic facies, provides the evaluation on the hydrocarbon potential in the Huizhou sag and can be applied as the basis for exploration.

\section{REFERENCES}

1. Meyer B L,Nederlof M H.: Identification of s-ource rocks on wireline logs by density/resis-tivity and sonic transittime/ resistivity crossplo-ts[J].AAPG Bulletin.1984, 68:121-129.

2. Fertl W H,Chillnger G V.: Total organic car-bon content determined from well logs[J].SPE Formation Evaluation,1988,3(2):407-419.

3. Hester T C,Schmoker J W,Sahl H.: Log-deriv-ed regional source-rock characteristics of theWoodford shale, Anadarko Basin,Oklahoma [J]. U S Geological Survey,Bulletin1866D,1990:1-38.

4. Herrson S L,Letendre I,Dufour M.: Source ro-ck evaluation using geochemical information from wireline logs and cores[J]. AAPG Bulle-tin.1988,72:1007.

5. Mann U P,Muller J.Source rock evaluation b-y well log analysis (Lower Toarcian,Hils Synli-ne).: Advances in organic geochemistry 1987[J]. Organic Geochemistry,1988,13:109-129.

6. BEERS R F.: Radioactivity and organic cont-ent of some Paleozoic shales[J]. AAPG Bulle-tin,1945,29(1):1-22.
7. Schmoker J W.: Determination of organic co-ntent of appalach-I an devonian shales from formation-density $\operatorname{logs}[J]$. AAPG Bulletin,1979,63:1504-1537.

8. Schmoker J W.: Determination of organicmat-ter content of appalachian devonian shale fr-om gammaray logs[J]. AAPG Bulletin,1981,65:1285-1298.

9. Schmoker J W,Hester T C.: Organic carboni-n bakken forma-tion, united states portion ofeil-liston basin[J].AAPG Bulle-tin,1983,67:2165-2174.

10. Herron S L.: A total organic carbon log for source rock evaluation[J].The Log Analyst,1987,28(6):520-527.

11. Passey Q R,Creaney S,Kulla J B,et al.: A pr-actical model for organic richness from poro-sity and resistivitylogs[J]. AAPG Bulletin,1990,74(12):1777-1794.

12. Mohammad Reza Kamalia,Ahad Allah Mirsha-dy.: Total or-ganic carbon content determine-d from well logs using $\Delta \log R$ and Neuro Fu-zzy techniques[J].Journal of Petroleum Scienc-e and Engineering,2004,45:141-148.

13. Lim J S.: Reservoir properties determination using fuzzy logic and neural networks[J]. Jou-rnal of Petroleum Science and Engineering,2005,49:182-192.

14. FERTL W H, RIEKE H H.: Gammaray spec-tral evaluation techniques identify fractured s-hale reservoirs and source rock characteristi-cs[J]. Journal of Petroleum Technology,1980,31(11):2053-2062.

15. Gong Zai sheng, Li Si tian.: Dynamic Resear-ch of Oil and Gas Accumulation in Norther-n Marginal Basins of South Chin a Sea[M].Beijing: Scien ce Press, 2004: 9-25.

16. Zeng Hongliu,Charise K.: Amplitude versus fr-equence-application to seismic stratigraghy a-nd reservoir characterization[A].Society of E-xploration Geophysicists,International Expositi-on and Seventieth Annual Meeting [C].Calga-ry,2000(8),6-11.

17. Simon Haykin.: Neural networks:a comprehen-sive Foundation.Second edition,1999.

18. Zeng Hongliu,Charise Kerans.: Amplitude vers-us frequency-applications to seismic stratigra-phy and reservoir characterization.SEG,2000.

19. Robinson E A.: Predictive decomposition of $t$-ime series with application to seismic explor-ation.Geophysics, 1954,32:418-484.

20. Robinson E A.: Predictive decomposition of s-eismic traces. Geophysics, 1957,22:767-778. 
21. Robinson E A, Treitel S.: Geophysical SignalAnalysis. Prentice-Hall, Inc, 1980.

22. Saggaf MM, Robinson E A.: A unified frame-work for the deconvolution of traces of non-white reflectivity.Geophysics, 2000,65:1660-1676.

23. Fu L Y. Joint lithologic inversion. In: WongP, Aminzadeh F,Nikravesh M eds.: Soft Com-puting for Reservoir Characterization and Mo-deling. Springer-Verlag Publishers, 2002. 511-530.

24. Fu L Y.: Joint inversions of seismic data foracoustic impedance.Geophysics, 2004,69: 994-1004.

25. Russell B H.: The application of multivariatestatistics and neural networks to the predicti-on of reservoir parameters using seismic attri-butes[D].Calgary:Department of Geology andGeophysics,Canada,2004.

26. Chopra S,Blias E,Manerikar A,et al.: Simultan-eous acquisition of $3 D$ VSP data-processing and intergration[J]. SEG Technical Program E-xpanded Abstracts,Society of Exploration Ge-ophysicists,2002,21:2337-2340.

\title{
CONTACT WITH AUTHOR
}

\author{
Wei Yang \\ e-mail:87390538@qq.com \\ tel.:15680051268
}

School of Geoscience and Technology

Southwest Petroleum University

Chengdu

China 